\title{
Beschaffungskonflikte: Volkswagen und seine Zulieferer
}

Ein Streit zwischen Volkswagen und zwei Zuliefererunternehmen der Gruppe Prevent sorgte dafür, dass mehr als $27700 \mathrm{VW}$-Mitarbeiter nicht planmäßig ihrer Arbeit nachgehen konnten und der VW-Konzern Kurzarbeit beantragte. Auslöser für den Konflikt war der von VW veranlasste Abbruch eines Entwicklungsauftrags mit dem Sitzehersteller Car Trim. Car Trim forderte Ausgleichszahlungen für bereits angefallene Kosten, Volkswagen hingegen wies diese Forderungen als überzogen zurück. Der Konflikt eskalierte, als Car Trims Schwesterfirma ES Automobilguss keine Getriebeteile mehr an VW lieferte und somit die Produktion der VW-Modelle Golf und Passat in sechs VW-Werken zum Erliegen brachte. Auch wenn die Streitparteien inzwischen eine Einigung erzielen konnten, bleibt doch ein ungutes Gefühl: Ist Kurzarbeitergeld, das von der Bundesagentur für Arbeit bezahlt wird, ein Instrument, das bei solchen Konflikten zum Einsatz kommen sollte? Und wie kann es sein, dass ein Konflikt mit einem Zulieferer einen Großkonzern zum Stillstand bringt?

Der Konflikt zwischen VW und Prevent ist nicht zuletzt auch wegen der von VW angeordneten Kurzarbeit und der damit verbundenen beantragten Zahlung von Kurzarbeitergeld durch die Bundesagentur für Arbeit in den Fokus der Öffentlichkeit gerückt. Die mögliche Einführung von Kurzarbeitszeit führt dazu, dass die Konfliktkosten auch von Arbeitnehmern und Steuerzahlern getragen werden und dadurch für die Konfliktparteien gesenkt werden. Das macht die Eskalation des Konflikts wahrscheinlicher und die Dauer länger. Es ist also fraglich, ob Kurzarbeitergeld in solchen Fällen gewährt werden sollte. Vor allem, weil ein solcher Konflikt den Steuerzahler nicht nur durch das Kurzarbeitergeld, sondern auch durch Steuerausfälle belastet.

Es ist umstritten, ob die Beantragung von Kurzarbeitergeld auf Kosten der Arbeitnehmer in diesem Fall rechtlich gedeckt ist. Die Voraussetzungen dafür sind, dass der Arbeitsausfall auf wirtschaftlichen Gründen oder auf einem unabwendbaren Ereignis beruht, dass der Arbeitsausfall unvermeidbar und nur vorübergehend ist. Da Prevent im vorliegenden Fall bestehende Verträge gebrochen hat, um den Konflikt zuzuspitzen und die Einhaltung von Verträgen ein schützenswertes Gut darstellt, kann die Beantragung von Kurzarbeitergeld als gerechtfertigt angesehen werden. Es wäre dennoch wünschenswert, dass bei der Formulierung der Kriterien für Kurzarbeitergeld die Auswirkungen auf die Konfliktwahrscheinlichkeit berücksichtigt würden.

Uneinigkeiten zwischen Lieferanten und Automobilherstellern sind nicht ungewöhnlich. Dass das Verhältnis zwischen Automobilherstellern und Zulieferern durchaus Konfliktpotenzial aufweist, macht nicht nur der vorliegende Fall deutlich. Außergewöhnlich am VW-Fall ist allerdings, dass ein Streit so eskaliert, dass er zu einem Lieferstopp führt. Mit einem solchen Lieferstopp riskiert der Zulieferer seine Reputation und sorgt durch den daraus resultierenden Produktionsstillstand beim Autohersteller für hohe Kosten. Es ist schon fast 20 Jahre her, dass zum letzten Mal in Deutschland die Bänder bei einem Automobilhersteller stillstanden, weil dieser mit einem Zulieferer im Streit lag: Im Jahr 1998 stellte der Türschlosshersteller Kiekert die Belieferung Fords im Zuge von Vertragsverhandlungen ein und verursachte dadurch einen Schaden von rund 100 Mio. D-Mark.

Im Automobilsektor stammen etwa 70\% der Komponenten von Zulieferern. Das bedeutet, dass sich Automobilhersteller nicht darauf beschränken, Standardteile wie Scheibenwischer und Reifen zuzukaufen. Vielmehr betrauen die Automobilherstel-

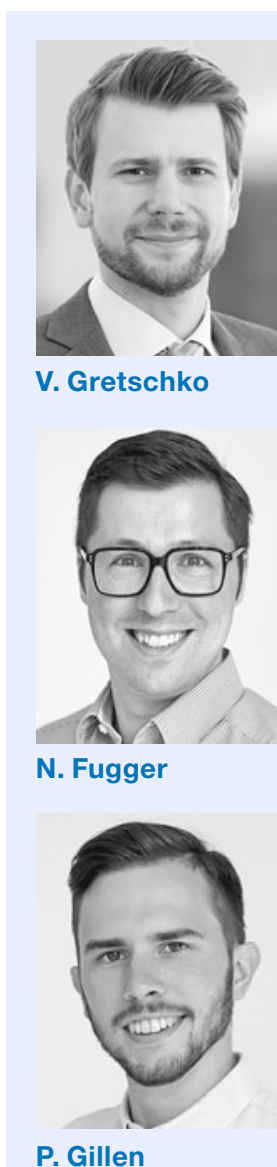

Vitali Gretschko ist Leiter der Forschungsgruppe Marktdesign am Zentrum für Europäische Wirtschaftsforschung in Mannheim.

Nicolas Fugger und Philippe Gillen sind Mitarbeiter in der Forschungsgruppe. 
ler Zulieferer auch mit der Entwicklung spezifischer Komponenten für die jeweiligen Fahrzeugmodelle. Der Einkauf solcher Entwicklungsleistungen und hochgradig individualisierter Komponenten von Zulieferern erfordert eine dauerhafte Zusammenarbeit, auf Langfristigkeit ausgelegte Verträge und beziehungsspezifische Investitionen auf Seiten des Zulieferers. Die Notwendigkeit der langfristigen Zusammenarbeit wird vor allem deutlich beim Blick auf die langen Produktzyklen in der Autobranche. So dauert die Entwicklung eines neuen Modells etwa drei bis vier Jahre. Anschließend ist das Fahrzeugmodell dann sechs bis acht Jahre auf dem Markt. Der lange Zeitraum sowie die technische Komplexität eines modernen Fahrzeugs erschweren die Gestaltung eines geeigneten Vertrags zwischen Automobilhersteller und Zulieferer. Jede nicht geklärte Eventualität, aber auch eine ungleiche Verteilung der Risiken auf die Vertragspartner kann zu starken Spannungen führen.

Dass ein einzelner Zulieferer in der Lage ist, durch einen Lieferstopp die Bänder bei einem Automobilhersteller zum Stillstand zu bringen, lässt sich nur durch die Kombination mehrerer Faktoren erklären. So sorgen die hochgradig individualisierten Komponenten und die eigens darauf abgestimmte Produktion der Zulieferer dafür, dass Zulieferer nicht kurzfristig ausgetauscht werden können. Hinzu kommt, dass viele Automobilhersteller gerade bei komplexeren Teilen dazu übergegangen sind, das Produkt von nur einem Lieferanten zu beziehen. Dieses sogenannte Single-Sourcing hat einerseits den Vorteil, dass es den Wettbewerb zwischen den Zulieferern intensiviert, die Ex-ante-Verhandlungsposition des Einkäufers stärkt und dadurch die Kosten reduziert. Andererseits erhöht es aber auch das Ausfallrisiko und bringt den Zulieferer in eine starke Ex-post-Verhandlungsposition. Letztlich wird die Gefahr eines Produktionsausfalls auch durch die anhaltende Entwicklung zu Just-in-time-Lieferungen und die damit verbundenen geringen Lagerbestände gesteigert.

Die Tatsache, dass der Lieferstopp eines einzelnen Zulieferers nicht nur ein einzelnes Fahrzeugmodell betrifft, ist darauf zurückzuführen, dass viele Automobilhersteller inzwischen eine Plattformstrategie verfolgen, bei der mehrere Modelle auf der gleichen Basis gebaut werden. Insbesondere VW setzt stark auf diese Art der Standardisierung und ist im Umkehrschluss anfälliger bei Ausfällen einzelner Bauteile.

Der vorliegende Fall zeigt, dass die optimale Gestaltung der Beziehungen zu den Zulieferern ein komplexes Problem darstellt. Ob ein Automobilhersteller das Ziel der langfristigen Gewinnmaximierung besser erreichen kann, wenn die Beziehung durch Wettbewerb anstelle von Kooperation geprägt ist, hängt letztlich von vielen Faktoren ab. Wenn die Leistung klar spezifiziert werden kann und Zulieferer leicht durch andere ersetzt werden können, ist Wettbewerb gut geeignet, um einen niedrigen Einkaufspreis zu erzielen. In Situationen, in denen Kooperation jedoch große Effizienzgewinne verspricht, kann es vorteilhaft sein, die Einkaufsstrategie in erster Linie darauf zu konzentrieren, die Effizienz zu steigern und sich erst nachgeordnet mit der Aufteilung der Effizienzgewinne zu beschäftigen. Beispielsweise hat Toyota, ein Pionier im Bereich des Just-in-time-Konzepts, mit der Kyohokai-Gruppe einen eigenen Zuliefererpool aufgebaut, in dem es nur wenig Fluktuation gibt und viele Unternehmensbeteiligungen seitens Toyotas. Solche Kooperationen haben allerdings auch gegenläufige Effekte. Sie reduzieren den Wettbewerb um die beste Idee und können Vetternwirtschaft begünstigen.

Welcher Weg der bessere ist, ist nicht eindeutig. Problematisch wird es aber, wenn Verhandlungsmacht und Wettbewerb in Strukturen angewendet werden, die auf Kooperation ausgerichtet sind. Dieses Spannungsfeld zwischen Wettbewerb und Kooperation macht Beschaffung zu einem komplexen und interessanten Forschungs- 